\title{
Bushen Zhuangjin decoction inhibits TM-induced chondrocyte apoptosis mediated by endoplasmic reticulum stress
}

\author{
PINGDONG LIN $^{1}$, XIAPING WENG ${ }^{1}$, FAYUAN LIU ${ }^{2}$, YUHUAN MA $^{1}$, HOUHUANG CHEN $^{2}$, \\ XIANG SHAO ${ }^{2}$, WENWEI ZHENG ${ }^{3}$, XIANXIANG LIU ${ }^{2}$, HONGZHI YE $^{1}$ and XIHAI LI ${ }^{2}$ \\ ${ }^{1}$ College of Pharmacy, ${ }^{2}$ Academy of Integrative Medicine, ${ }^{3}$ Fujian Key Laboratory of Integrative Medicine on Geriatrics, \\ Fujian University of Traditional Chinese Medicine, Fuzhou, Fujian 350122, P.R. China
}

Received March 22, 2015; Accepted October 8, 2015

DOI: $10.3892 / \mathrm{ijmm} .2015 .2387$

\begin{abstract}
Chondrocyte apoptosis triggered by endoplasmic reticulum (ER) stress plays a vital role in the pathogenesis of osteoarthritis (OA). Bushen Zhuangjin decoction (BZD) has been widely used in the treatment of OA. However, the cellular and molecular mechanisms responsible for the inhibitory effects of BZD on chondrocyte apoptosis remain to be elucidated. In the present study, we investigated the effects of BZD on ER stress-induced chondrocyte apoptosis using a chondrocyte in vitro model of OA. Chondrocytes obtained from the articular cartilage of the knee joints of Sprague Dawley (SD) rats were detected by immunohistochemical staining for type II collagen. The ER stress-mediated apoptosis of tunicamycin (TM)-stimulated chondrocytes was detected using 4-phenylbutyric acid (4-PBA). We found that 4-PBA inhibited TM-induced chondrocyte apoptosis, which confirmed the successful induction of chondrocyte apoptosis. BZD enhanced the viability of the TM-stimulated chondrocytes in a dose- and time-dependent manner, as shown by MTT assay. The apoptotic rate and the loss of mitochondrial membrane potential $(\Delta \Psi \mathrm{m})$ of the TM-stimulated chondrocytes treated with BZD was markedly decreased compared with those of chondrocytes not treated with BZD, as shown by 4',6-diamidino-2-phenylindole (DAPI) staining, Annexin V-FITC binding assay and JC-1 assay. To further elucidate the mechanisms responsible for the inhibitory effects of BZD on TM-induced chondrocyte apoptosis mediated by ER stress, the mRNA and protein expression levels of binding immunoglobulin protein (Bip), X-box binding protein 1 (Xbpl), activating transcription factor 4 (Atf4), C/EBP-homologous
\end{abstract}

Correspondence to: Professor Hongzhi Ye, College of Pharmacy, Fujian University of Traditional Chinese Medicine, 1 Qiuyang Road, Minhou, Shangjie, Fuzhou, Fujian 350122, P.R. China

E-mail: yelin0930@163.com

Professor Xihai Li, Academy of Integrative Medicine, Fujian University of Traditional Chinese Medicine, 1 Qiuyang Road, Minhou Shangjie, Fuzhou, Fujian 350122, P.R. China

E-mail: lixihai79dahai@163.com

Key words: osteoarthritis, chondrocytes, apoptosis, endoplasmic reticulum stress, Bushen Zhuangjin decoction protein (Chop), caspase-9, caspase-3, B-cell lymphoma 2 (Bcl-2) and $\mathrm{Bcl}-2$-associated $\mathrm{X}$ protein (Bax) were measured by reverse transcription-polymerase chain reaction (RT-PCR) and western blot analysis. In the TM-stimulated chondrocytes treated with BZD, the mRNA and protein expression levels of Bip, Atf4, Chop, caspase-9, caspase-3 and Bax were significantly decreased, whereas the mRNA and protein expression levels of Xbpl and Bcl-2 were significantly increased compared with the TM-stimulated chondrocytes not treated with BZD. Additionally, all our findings demonstrated that there was no significant difference between the TM-stimulated chondrocytes treated with BZD and those treated with 4-PBA. Taken together, our results indicate that BZD inhibits TM-induced chondrocyte apoptosis mediated by ER stress. Thus, BZD may be a potential therapeutic agent for use in the treatment of OA.

\section{Introduction}

Osteoarthritis (OA), a cause of disability, is characterized by changes in chondrocyte gene expression and extracellular matrix (ECM) destruction $(1,2)$. Chondrocytes are the only cell type present in articular cartilage, and their biosynthetic activities are necessary to maintain the stability of the cartilage. Several lines of evidence indicate that chondrocyte death is a key player in cartilage degeneration (3-5). Chondrocyte death may occur due to apoptosis; it is therefore possible that chondrocyte loss caused by apoptosis plays a crucial role in the pathogenesis of OA $(6,7)$. Recent studies have also demonstrated that endoplasmic reticulum (ER) stress, upstream of the mitochondria-dependent apoptotic signal transduction pathway, plays a role in chondrocyte apoptosis $(8,9)$.

ER stress, as a new apoptotic pathway, results from an imbalance between the load of unfolded proteins in the ER and the capacity of the ER (10). It is considered that there are three apoptotic pathways triggered by ER stress: the C/EBP-homologous protein (Chop)/growth arrest and DNA damage-inducible gene 153 (Gadd153) pathway, the c-JUN $\mathrm{NH}_{2}$-terminal kinase (JNK) pathway and the caspase pathway (11). The transcription of the Chop gene is mediated by three signal transmission passages: PKR-like ER kinase (PERK), activating transcription factor (Atf) 6 and inositol-requiring protein 1 (IRE1) (12). The three protein molecules activate the apoptotic genes, Gadd153 and TRAF2 
(the gene for the activation of JNK), and initiate chondrocyte apoptosis $(12,13)$. In addition, the apoptotic genes, caspase-3 and caspase-9, are activated through the caspase pathway, thereby executing the final stages of apoptosis (14). Several critical signaling molecules, such as binding immunoglobulin protein (Bip), X-box binding protein 1 (Xbpl), Atf4, Bcl-2associated $\mathrm{X}$ protein (Bax) and B-cell lymphoma 2 (Bcl-2) have been shown to participate in the process of apoptosis induced by ER stress (15). Thus, decreasing apoptosis through by regulating the effects of related signaling molecules is crucial to preventing the progressive degeneration of articular cartilage.

Bushen Zhuangjin decoction (BZD) is a well-known formulation that nourishes the Gan (liver) and the Shen (kidney) and also strengthens tendons and bones, and has been long used in China (16). Clinical studies have demonstrated that BZD has a positive effect on the treatment of $\mathrm{OA}$, and experimental studies have found that BZD decreases the apoptosis of chondrocytes and promotes chondrocyte proliferation $(17,18)$. However, the specific mechanisms responsible for the inhibitory effects of BZD on ER stress-mediated chondrocyte apoptosis remain to be elucidated. Therefore, in this study, we aimed to determine whether BZD inhibits the tunicamycin (TM)-induced apoptosis of chondrocytes mediated by ER stress and to elucidate the underlying mechanisms.

\section{Materials and methods}

Preparation of the BZD aqueous extract. BZD consists of the following 10 herbs: $12 \mathrm{~g}$ Shu Di Huang (steamed Chinese foxglove, Radix Rehmanniae Glutinosae Conquitae), $10 \mathrm{~g}$ Niu Xi (Achyranthes bidentata), 12 g Dang Gui (Angelica sinensis), $12 \mathrm{~g} \mathrm{Fu}$ Ling (Poria cocos), $12 \mathrm{~g} \mathrm{Xu}$ Duan (Dipsacus), $12 \mathrm{~g}$ Shan Zhu Yu (Cornus officinalis), $10 \mathrm{~g}$ Bai Shao (white peony root, Radix Paeoniae Alba), $10 \mathrm{~g}$ Du Zhong (Eucommia bark), $10 \mathrm{~g} \mathrm{Wu} \mathrm{Jia} \mathrm{Pi} \mathrm{(Acanthopanax} \mathrm{root} \mathrm{bark)}$ and $5 \mathrm{~g}$ Qing Pi (immature tangerine peel, Pericarpium Citri Reticulatae Viride). All herbs were purchased from the Third People's Hospital of Fujian University of Traditional Chinese Medicine (FJUTCM; Fuzhou, China) and were identified by the Teaching and Research section of FJUTCM. The components were mixed and extracted with standard methods according to Chinese Pharmacopoeia (Chinese Pharmacopoeia Committee, 2010). The herbs of BZD were extracted with distilled water by a refluxing method and were then filtered and concentrated. The filtrate of BZD was evaporated using a rotary evaporator (model RE-2000; Shanghai Yarong Biochemistry Instrument Factory, Shanghai, China) and was then dried to a constant weight with a vacuum drying oven (model DZF-300; Shanghai Yiheng Scientific Instrument Co., Ltd., Shanghai, China). The water extract of BZD was finally dissolved in phosphate-buffered saline (PBS; HyClone Laboratories, Inc., Logan, UT, USA) to the concentration of $10 \mathrm{mg} / \mathrm{ml}$ and was stored at $-20^{\circ} \mathrm{C}$. The working concentrations of BZD were prepared by diluting the stock solution in Dulbecco's modified Eagle's medium (DMEM) containing 10\% fetal bovine serum (FBS) (both from HyClone Laboratories, Inc.) and then by filtering it through a $0.22-\mu \mathrm{m}$ filter and storing it at $4^{\circ} \mathrm{C}$.

Chondrocyte culture and identification. Articular chondrocytes were isolated and cultured as previously described (19). In brief,
$4 \mathrm{SD}$ rats were sacrificed by cervical dislocation. The articular cartilage was cut out from the bilateral knee joints, peeled off under sterile conditions and rinsed with PBS (HyClone Laboratories, Inc.) 3 times. The cartilage was cut into sections of $1 \mathrm{~mm}^{3}$ and digested with $0.2 \%$ collagenase II (Sigma-Aldrich, St. Louis, MO, USA) in a $37^{\circ} \mathrm{C} 5 \% \mathrm{CO}^{2}$ incubator. The supernatant fluid was collected every $90 \mathrm{~min}$, and centrifuged for $5 \mathrm{~min}(1,000 \mathrm{rpm} / \mathrm{min})$. The precipitate was resuspended with DMEM containing 10\% FBS (both from HyClone Laboratories, Inc.) and transferred onto a $25 \mathrm{~mm}^{2}$ culture flask and cultured in a $37^{\circ} \mathrm{C} 5 \% \mathrm{CO}_{2}$ incubator; these were termed the primary chondrocytes. The Sprague Dawley (SD) rats used in this study were purchased from Shanghai SLAC Laboratory Animal Co., Ltd. (Shanghai, China). The present study was approved by the Institutional Animal Care and Use Committee of FJUTCM. The morphological changes and growth characteristics of the chondrocytes were recorded under a phase contrast microscope (Olympus, Tokyo, Japan). To identify the chondrocytes, immunocytochemical staining for type II collagen was performed. Briefly, the cells were seeded onto sterilized coverslips and placed into wells of a 12 -well plate $\left(2 \times 10^{5}\right.$ cells/ well), and cultured in a $37^{\circ} \mathrm{C} 5 \% \mathrm{CO}_{2}$ incubator for $48 \mathrm{~h}$. The cells were fixed with $4 \%$ paraformaldehyde (Sigma-Aldrich) at $4^{\circ} \mathrm{C}$ for $30 \mathrm{~min}$, and blocked with $10 \%$ bovine serum albumin (BSA; Sigma-Aldrich) for $1 \mathrm{~h}$. The slides were incubated with a rabbit polyclonal antibody to type II collagen (BS1071; Bioworld Technology, Inc., St. Louis Park, MN, USA) overnight at $4^{\circ} \mathrm{C}$. The slides were then incubated with peroxidase-conjugated affinipure goat anti-rabbit IgG (ZB-2301; Zhongshan GoldenBridge Biotechnology, Co., Ltd., Beijing, China) for $30 \mathrm{~min}$, followed by incubation with diaminobenzidine (ZLI-9018 DAB kit; Zhongshan GoldenBridge Biotechnology, Co., Ltd.) for $5 \mathrm{~min}$. The slides were finaly counterstained with hematoxylin (SigmaAldrich) and dehydrated. Images were captured using a light microscope (BH2; Olympus, Tokyo, Japan). The primary chondrocytes were termed passage $(\mathrm{P}) 0$ and $\mathrm{P} 2$ chondrocytes at approximately $80 \%$ confluence were used in our experiments.

Cell viability assay. The chondrocytes were seeded in 96-well culture plates at a density of $5 \times 10^{4}$ cells $/ \mathrm{ml}$ and incubated (100 $\mu \mathrm{l} /$ well) for $24 \mathrm{~h}$. The chondrocytes were stimulated with $2 \mu \mathrm{g} / \mathrm{ml}$ TM (Sigma-Aldrich) as the model group. A series of concentrations of BZD $(50,100,200,400,800,1200 \mu \mathrm{g} / \mathrm{ml})$ were also added in the presence of TM follwed by incubation for 24, 48 or $72 \mathrm{~h}$. Following intervention, $100 \mu \mathrm{l}$ 1\% MTT (Sigma-Aldrich) were added to each well followed by incubation at $37^{\circ} \mathrm{C}$ for $4 \mathrm{~h}$, and the supernatant was then replaced with $150 \mu \mathrm{l} /$ well of DMSO (Hengxing Chemical Preparation Co., Ltd., Tianjin, China) and shaken for $10 \mathrm{~min}$. The absorbance was detected using an ELISA reader (model ELX800; BioTek Instruments, Inc., Winooski, VT, USA) by measuring the optical denstiy (OD) value at $490 \mathrm{~nm}$.

Experimental design. According to the assay, there were 4 experimental groups as follows: the control group, which included P2 chondrocytes without treatment; the model group, which included apoptotic chondrocytes stimulated with TM; the dosing group, which included TM-stimulated chondrocytes treated with BZD; and the positive control group, which included TM-stimulated chondrocytes treated with $5 \mathrm{mM} 4$-phenylbutyric acid (4-PBA) (Sigma-Aldrich), as previously described (20). 

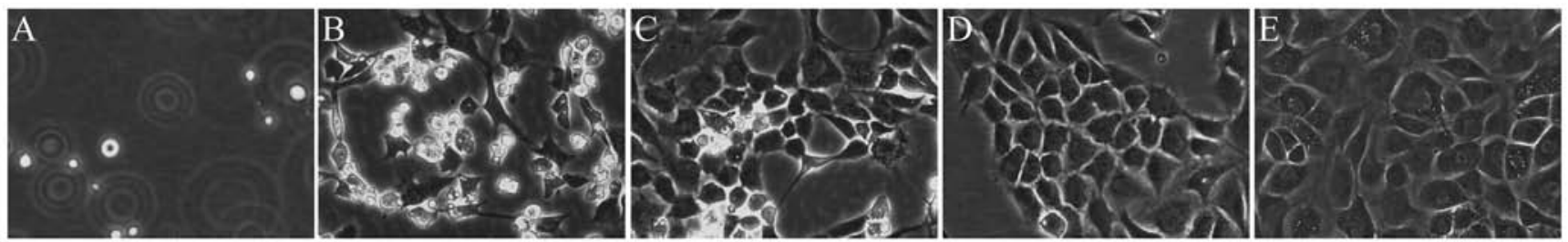

Figure 1. Morphology of chondrocytes (magnification, x200). (A) Newly isolated chondrocytes. (B) Primary cells cultured for 3 days. (C) Primary cells cultured for 6 days. (D) First-passage cells cultured for 3 days. (E) Second-passage cells cultured for 3 days.

4',6-Diamidino-2-phenylindole (DAPI) staining. The chondrocytes were washed with PBS and fixed with $4 \%$ paraformaldehyde (Sigma-Aldrich) at $4^{\circ} \mathrm{C}$ for $15 \mathrm{~min}$. The chondrocytes were stained in $5 \mu \mathrm{g} / \mathrm{ml}$ DAPI for $5 \mathrm{~min}$. The images of apoptotic chondrocyte morphology were captured using a fluorescence microscope (Olympus).

Detection of apoptosis by flow cytometry with Annexin V-FITC and JC-1 staining. The apoptotic rate of the chondrocytes was detected with Annexin V/PI staining (Nanjing KeyGen Biotech, Nanjing, China) using a fluorescence-activated cell sorting (FACS) machine (FACSCalibur ${ }^{\mathrm{TM}}$; Becton-Dicskinson Biosciences, San Diego, CA, USA). The changes in mitochondrial membrane potential $(\Delta \Psi \mathrm{m})$ were measured using a JC-1 kit (Nanjing Keygen Biotech), and the processed cells were also analyzed using the FACS machine. Both staining procedures were carried out according to the relevant manufacturer's instructions.

Reverse transcription-polymerase chain reaction $(R T-P C R)$. Total RNA was extracted from the cells using TRIzol reagent (Invitrogen Life Technologies, Grand Island, NY, USA). RNA $(1 \mu \mathrm{g})$ was reverse transcribed into cDNA using a reverse transcription kit (Thermo Fisher Scientific, Inc., Waltham, MA, USA) according to the manufacturer's instructions. Subsequently, the DNA bands were analyzed by gel electrophoresis (1.5\% agarose) using a gel documentation system (Bio-Rad, Hercules, CA, USA) and normalized to those of $\beta$-actin. The PCR primers used were as follows: $\beta$-actin forward, 5'-GAG AGG GAA ATC GTG CGT GAC-3' and reverse, 5'-CAT CTG CTG GAA GGT GGA CA-3'; Bip forward, 5'-ATC AAC CCA GAT GAG GCT GTA GCA-3' and reverse, 5'-AGA CCT TGA TTG TTA CGG TGG GCT-3'; Xbp1 forward, 5'-AGC ATA GGC CTG TCT GCT TCA CTA-3' and reverse, 5'-TGG TAA AGT CCA GCA CTT GGG AGT-3'; Atf4 forward, 5'-AAT GGC TGG CTA TGG ATG GG-3' and reverse, 5'-TGT CTG AGG GGG CTC CTT ATT AG-3'; caspase-9 forward, 5'-GCC TCA TCATCA ACA ACG-3' and reverse, 5'-CTG GTA TGG GAC AGC ATC T-3'; caspase-3 forward, 5'-GGA CCT GTG GAC CTG AAA-3' and reverse, 5'-GGG TGC GGT AGA GTA AGC-3'; Bcl-2 forward, 5'-TGG CAT CTT CTC CTT CCC-3' and reverse, 5'-GGT ACA TCT CCC TGT TGA CG-3'; Chop forward, 5'-TTC ACT ACT CTT GAC CCT GCG TC-3' and reverse, 5'-CAC TGA CCA CTC TGT TTC CGT TTC-3'; Bax forward, 5'-GGC GAT GAA CTG GAC AAC-3' and reverse, 5'-TCC CGA AGT AGG AAA GGA G-3'.

Western blot analysis. Total protein was extracted from the cells using radioimmunoprecipitation assay (RIPA) lysis buffer (Beyotime Biotech, Shanghai, China) with $1 \mathrm{mM}$ phenylmethanesulfonyl fluoride (PMSF; Beyotime Biotech), and the bicinchoninic acid assay (BCA) was used to measure the protein concentrations. Proteins were separated by electrophoresis on SDS-PAGE gels (12\%) and electrotransferred onto PVDF membranes (Thermo Fisher Scientific, Inc.). The membranes were blocked in 5\% skimmed milk, then incubated with primary antibodies against Bip (3183S), Xbp1 (12782), Atf4 (11815S), Chop (5554S), Bcl-2 (2876S), caspase-9 (9506S), caspase-3 (9662S) and Bax (2772S; all from Cell Signaling Technology, Inc., Beverly, MA, USA) and $\beta$-actin (sc-47778; Santa Cruz Biotechnology, Inc., Santa Cruz, CA, USA) at $4^{\circ} \mathrm{C}$ overnight, followed by goat anti-rabbit horseradish peroxidase-conjugated (HRP) secondary antibody IgG (ZB-2301; Zhongshan GoldenBridge Biotechnology, Co., Ltd.) at room temperature for $1 \mathrm{~h}$. Eventually, the blots were detected by using a Bio-Rad ChemiDoc XRS+ imaging system (Bio-Rad), and $\beta$-actin was used as the internal control.

Statistical analysis. Data were analyzed by one-way analysis of variance (ANOVA) or the Student's t-test using SPSS 19.0 software and all values are expressed as the means \pm SD. A value of $\mathrm{P}<0.01$ was considered to indicate a statistically significant difference. A value of $\mathrm{P}<0.05$ was considered a trend.

\section{Results}

Morphology and identification of chondrocytes. The chondrocytes in our study exhibited a morphology which is considered typical of chondrocytes, with a spherical, fusiform and slabstone shape (Fig. 1), as described in previous studies $(21,22)$. Immunohistochemical staining for type II collagen indicated that the cytoplasm was stained brown, which represented the positive expression of type II collagen, while no brown staining was observed in the negative control cells (Fig. 2). Following toluidine blue staining, red/purple particles in the cytoplasm were observed under a light microscope, which indicated the staining of proteoglycans (23) (Fig. 2). Thus, P2 chondrocytes were used in our experiments, as they were proven to be rich in ECM components (type II collagen) and had the typical morphology of chondrocytes.

BZD enhances the viability of chondrocytes stimulated with $T M$. To examine the effects of BZD on chondrocyte viability, the viability of the TM-stimulated chondrocytes treated with BZD at various concentrations for different periods of time was detected by MTT assay. The viability of the TM-stimulated chondrocytes was markedly lower than that of the untreated cells in the control group $(\mathrm{P}<0.01)$. The viability of the 

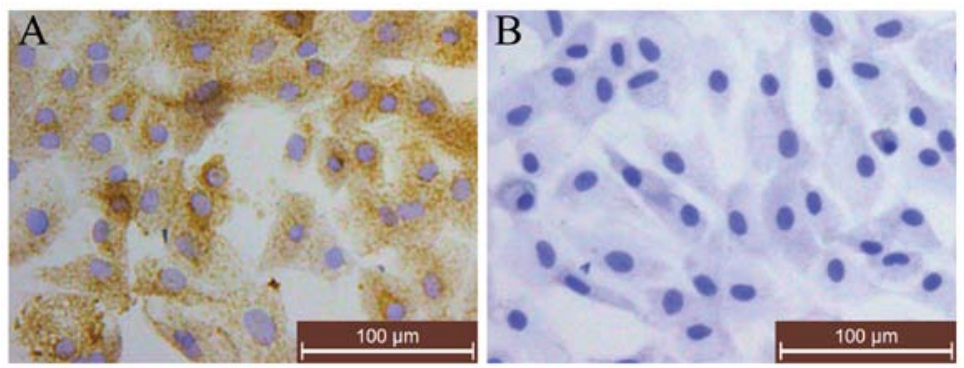

Figure 2. Chondrocyte identification by immunohistochemical stainaing for type II collagen (magnification, x200). (A) Positive P2 chondrocytes stained brown in the cytoplasm. (B) P2 chondrocytes negative for type II collagen did not stain.
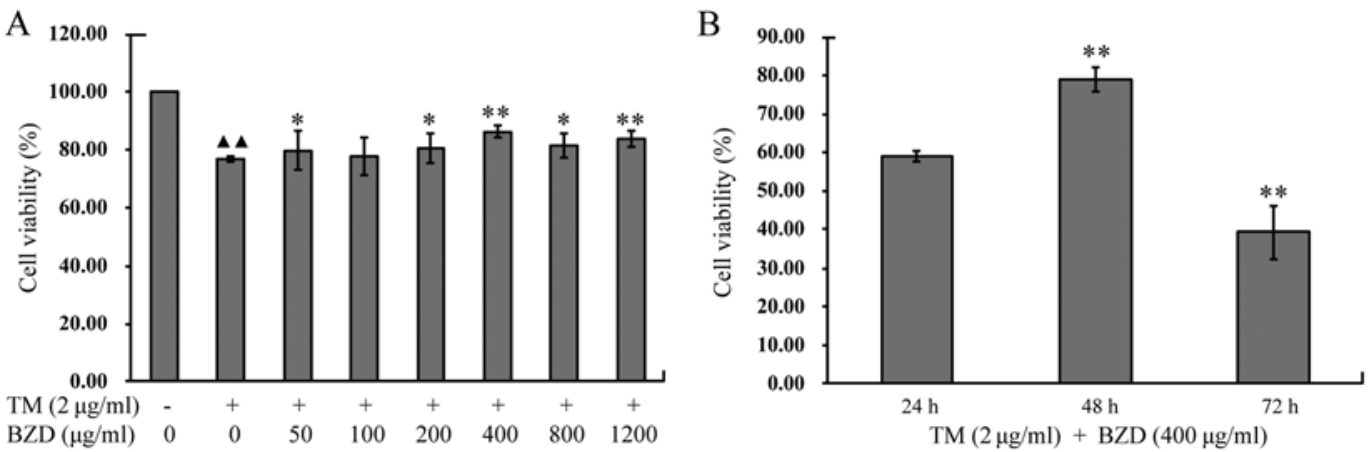

Figure 3. Bushen Zhuangjin decoction (BZD) enhances the viability of tunicamycin (TM)-stimulated chondrocytes. (A) Chondrocytes treated with TM and BZD at various concentrations for $24 \mathrm{~h}$. Values are means $\pm \mathrm{SD}$ (vertical bars); ${ }^{\wedge} \mathrm{P}<0.01$ compared to untreated cells (not treated with either TM or BZD); ${ }^{* *} \mathrm{P}<0.01$, ${ }^{*} \mathrm{P}<0.05$ compared to TM-stimulated chondrocytes. (B) Viability of TM-stimulated chondrocytes treated with $400 \mu \mathrm{g} / \mathrm{ml} \mathrm{BZD} \mathrm{for} \mathrm{various} \mathrm{periods} \mathrm{of} \mathrm{time.}$ Values are the means $\pm \mathrm{SD}$ (vertical bars), ${ }^{* *} \mathrm{P}<0.01$ and ${ }^{*} \mathrm{P}<0.05$, compared to $24 \mathrm{~h}$.

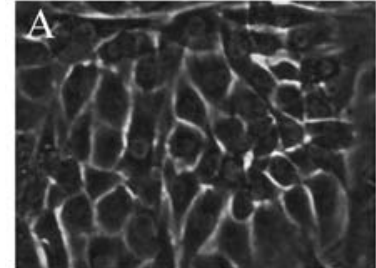

4-PBA $(5 \mathrm{mM})$

$\mathrm{TM}(2 \mu \mathrm{g} / \mathrm{ml}) \quad-$

$\mathrm{BZD}(\mu \mathrm{g} / \mathrm{ml}) \quad 0$

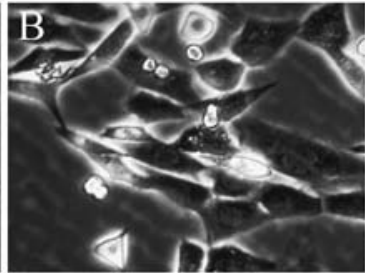

$-$

$+$

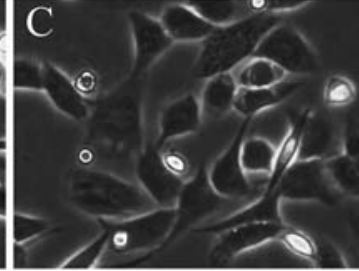

$+$

400

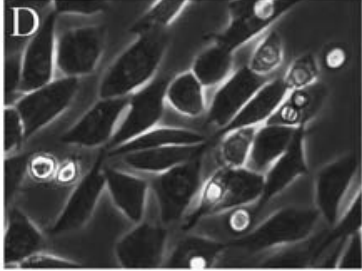

$+$

$+$

0

Figure 4. Bushen Zhuangjin decoction (BZD) prevents morphological changes of tunicamycin (TM)-stimulated chondrocytes (magnification, x200). (A) Untreated cells, the control group. (B) TM-stimulated chondrocytes, the model group. (C) The viability of TM-stimulated chondrocytes treated with $400 \mu \mathrm{g} / \mathrm{ml}$ of BZD for $48 \mathrm{~h}$, the dosing group. (D) The viability of TM-stimulated chondrocytes treated with $5 \mathrm{mM} 4$-phenylbutyric acid (4-PBA) for $20 \mathrm{~h}$, the positive control group.

TM-stimulated chondrocytes treated with various concentrations of BZD was enhanced compared with the untreated TM-stimulated chondrocytes $(\mathrm{P}<0.01$ or $\mathrm{P}<0.05$; Fig. $3 \mathrm{~A})$. The viability of the TM-stimulated chondrocytes treated with $400 \mu \mathrm{g} / \mathrm{ml}$ BZD for $48 \mathrm{~h}$ was markedly enhanced compared with that of the TM-stimulated chondrocytes treated with $400 \mu \mathrm{g} / \mathrm{ml}$ BZD for 24 or $72 \mathrm{~h}(\mathrm{P}<0.01$;Fig. 3B). These findings indicate that BZD exerts a positive effect on TM-stimulated chondrocytes in a dose- and time-dependent manner. Finally, the most suitable concentration $(400 \mu \mathrm{g} / \mathrm{ml})$ of BZD for $48 \mathrm{~h}$ of treatment was used in all the subsequent experiments.

Effects of BZD on the morphology of TM-stimulated chondrocytes. Following treatment, the morphological changes of the TM-stimulated chondrocytes were observed under a phase contrast microscope. It was observed that the untreated cells in the control group retained their normal growth state and exhibited no significant changes in morphology (Fig. 4A). However, the TM-stimulated chondrocytes became elongated and shrank in size, and were observed floating in the medium, and were also detached from each other (Fig.4B). However, these changes in cell morphology induced by stimulation with TM were not observed or were less evident in the TM-stimulated chondrocytes treated with BZD or 4-PBA than in the model group (Fig. 4C and D).

BZD decreases the apoptosis of TM-stimulated chondrocytes. DAPI staining was used to monitor cell apoptosis, by emitting a strong fluorescence signal when DAPI combined with DNA in the nucleus. Normal nuclei were round in shape and light blue in color. However, nuclei of the apoptotic cells were stained bright 


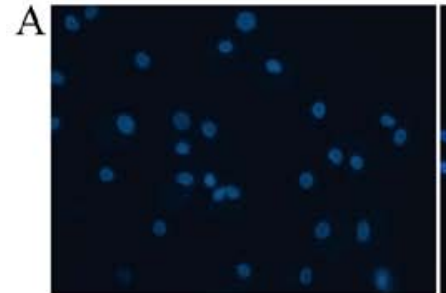

4-PBA (5 mM)

$\mathrm{TM}(2 \mu \mathrm{g} / \mathrm{ml})$

BZD $(\mu \mathrm{g} / \mathrm{ml})$
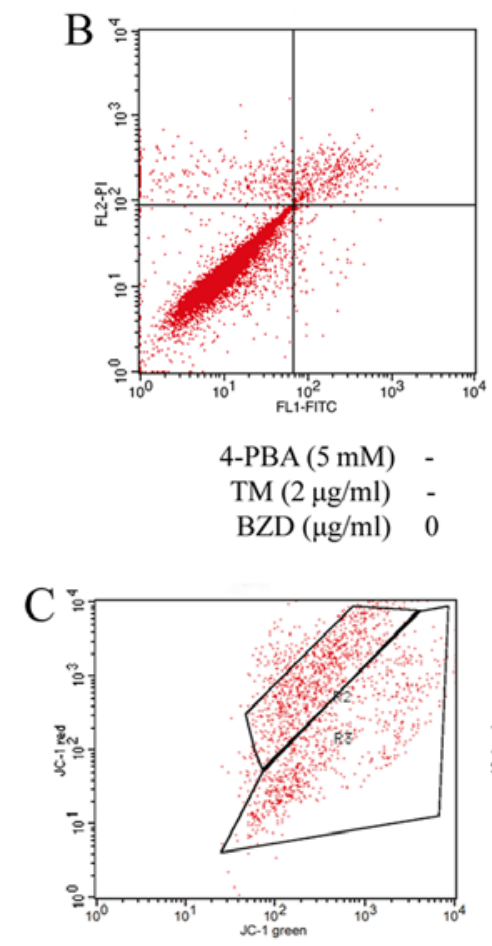

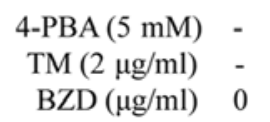

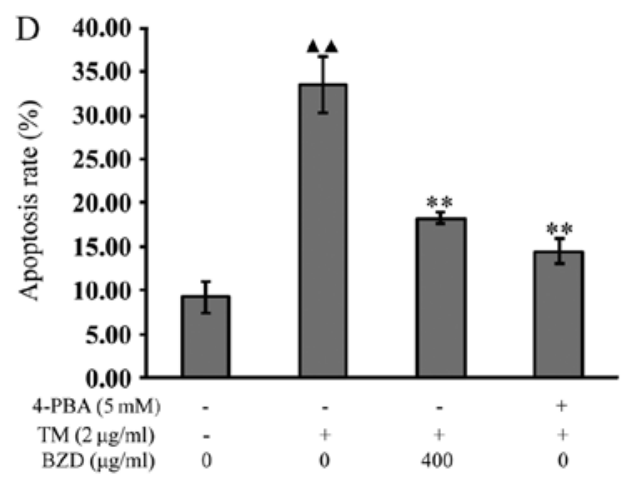

$+$

0

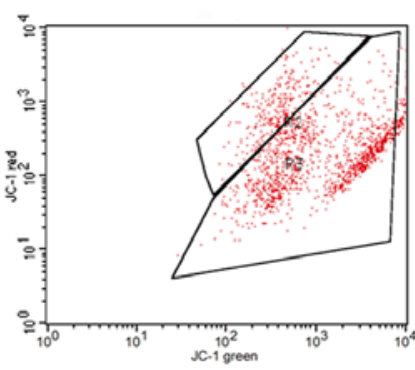

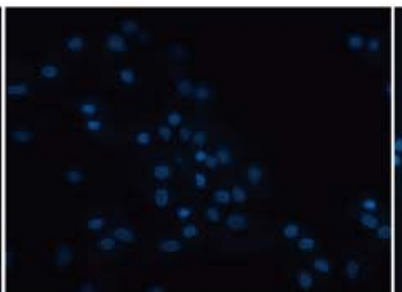

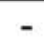

$+$

400
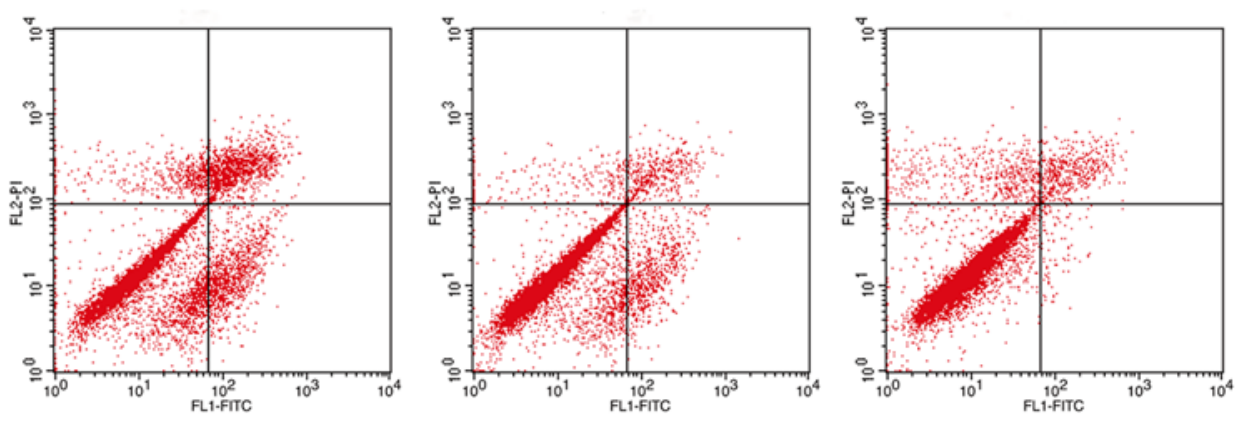

400
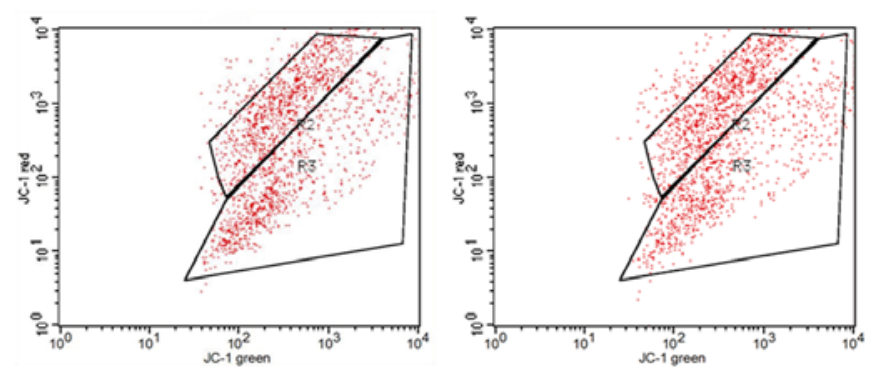

$+$

$+$

400

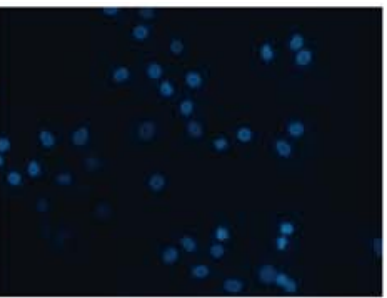

$+$

$+$

0

$+$

+
0

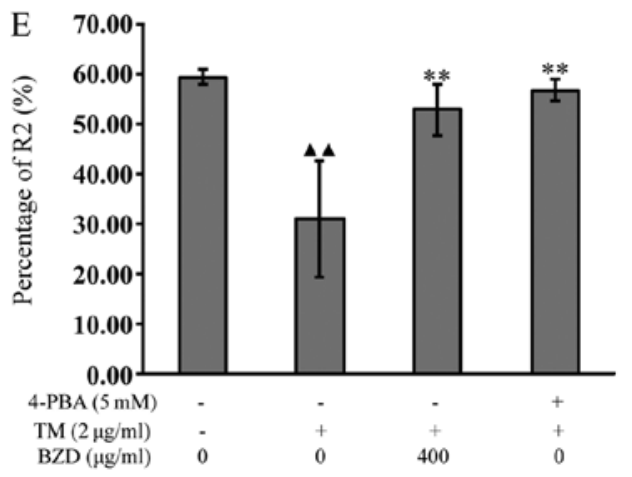

Figure 5. Bushen Zhuangjin decoction (BZD) inhibits the apoptosis and decrease in mitochondrial membrane potential ( $\Delta \Psi \mathrm{m})$ in tunicamycin (TM)-stimulated chondrocytes. (A) Morphological changes in chondrocytes associated with apoptosis were examined by 4',6-diamidino-2-phenylindole (DAPI) staining. (B) Chondrocyte apoptotic rate was assessed by Annexin V/propidium iodide (PI) staining. (C) The changes in $\Delta \Psi \mathrm{m}$ in chondrocytes treated with TM and BZD at various concentrations and chondrocytes left untreated were revealed by JC-1 staining. (D) Percentage of apoptotic cells in chondrocytes treated with TM and BZD at various concentrations and untreated chondrocytes. (E) Percentage of live cells (P2) in chondrocytes treated with TM and BZD at various concentrations and untreated chondrocytes. ${ }^{\wedge} \mathrm{P}<0.01$, compared to untreated cells; ${ }^{* *} \mathrm{P}<0.01$, compared to TM-stimulated chondrocytes. 4-PBA, 4-phenylbutyric acid.

blue and became deformed or fragmented due to the aggregation of DNA in the TM-stimulated cells. In the dosing and positive control groups, the apoptosis of the chondrocyte nuclei was still evident, but to a lesser extent than the model group (Fig. 5A). 


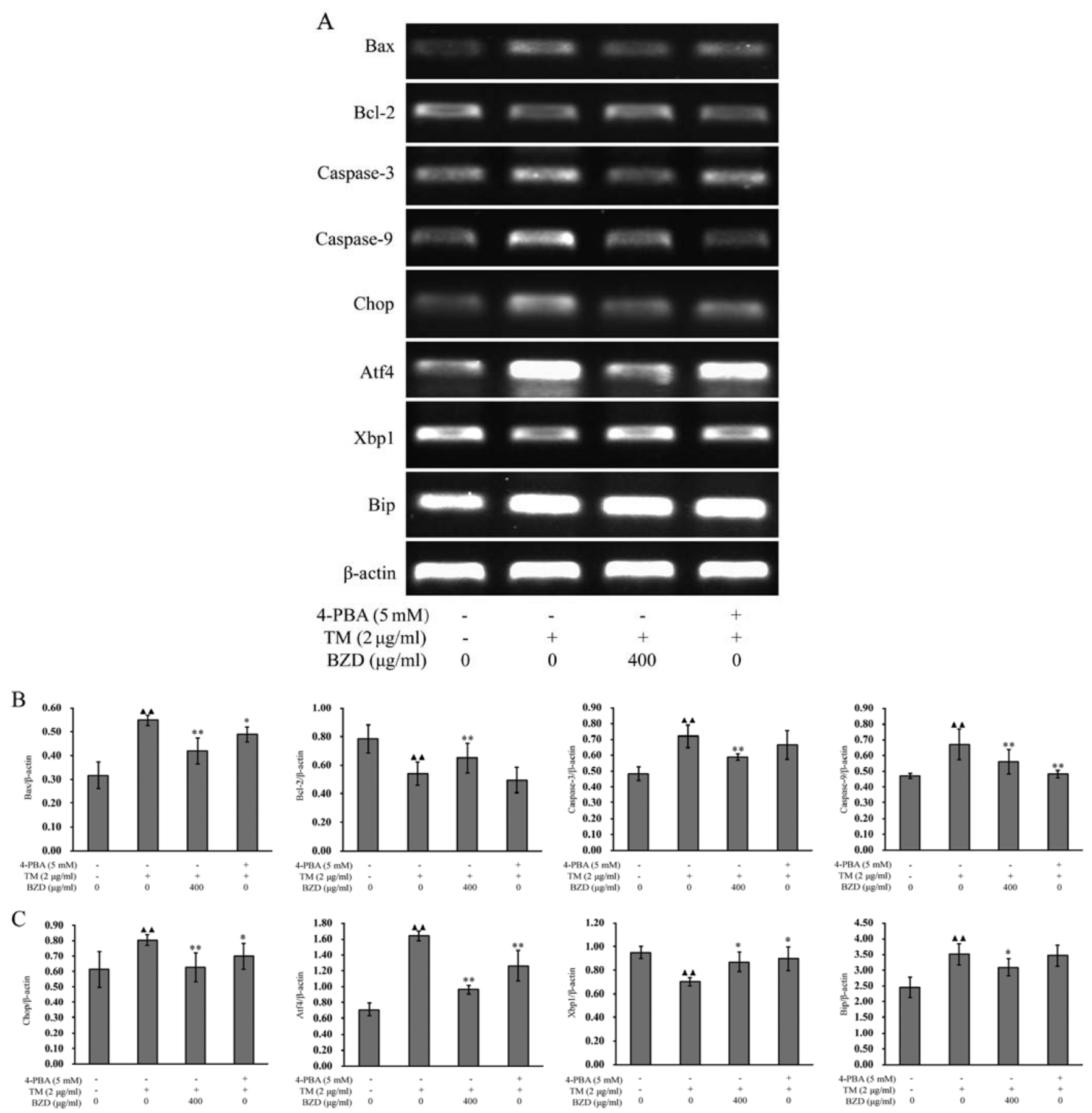

Figure 6. Bushen Zhuangjin decoction (BZD) prevents tunicamycin (TM)-induced chondrocyte apoptosis. (A-C) mRNA expression levels of apoptosis-related factros in chondrocytes left untreated and in those treated with TM and BZD or 4-phenylbutyric acid (4-PBA) in the presence of TM for $24 \mathrm{~h}$ were measured by reverse transcription-polymerase chain reaction (RT-PCR). We measured the mRNA expression levels of binding immunoglobin protein (Bip), X-box binding protein 1 (Xbp1), activation transcription factor 4 (Atf4), C/EBP-homologous protein (Chop), (B) Bcl-2-associated X protein (Bax), B-cell lymphoma 2 (Bcl-2), caspase-3 and caspase-9. $\beta$-actin was used as the internal control for normalization. Values are means $\pm \mathrm{SD}$ (vertical bars). ${ }^{\wedge} \mathrm{P}<0.01$ compared to untreated cells; ${ }^{* *} \mathrm{P}<0.01,{ }^{*} \mathrm{P}<0.05$ compared to TM-stimulated chondrocytes.

To further examine the effects of BZD on the apoptosis of TM-stimulated chondrocytes, Annexin V-FITC binding assay was used to measure chondrocyte apoptosis. As shown in Fig. 5B and D, the number of dead, apoptotic cells and the apoptotic rate of the TM-stimulated chondrocytes treated with BZD were significantly lower than those of the TM-stimulated cells not treated with BZD. The results revealed that BZD exerted a positive effect by reducing or inhibiting the apoptosis of TM-stimulated chondrocytes.
JC-1 is an indicator of the initiation of cell apoptosis that is widely used to quantify $\Delta \Psi \mathrm{m}$ by measuring the fluorescence intensities. The effects of BZD on the changes in $\Delta \Psi \mathrm{m}$ were examined by JC-1 assay. As shown in Fig. 5C and E, a significant reduction in $\Delta \Psi \mathrm{m}$ in the TM-stimulated chondrocytes was observed compared with the untreated cells. When compared with the TM-stimulated chondrocytes, the $\Delta \Psi \mathrm{m}$ in the TM-stimulated chondrocytes treated with BZD or 4-PBA was markedly increased. This suggests that BZD exerts a posi- 

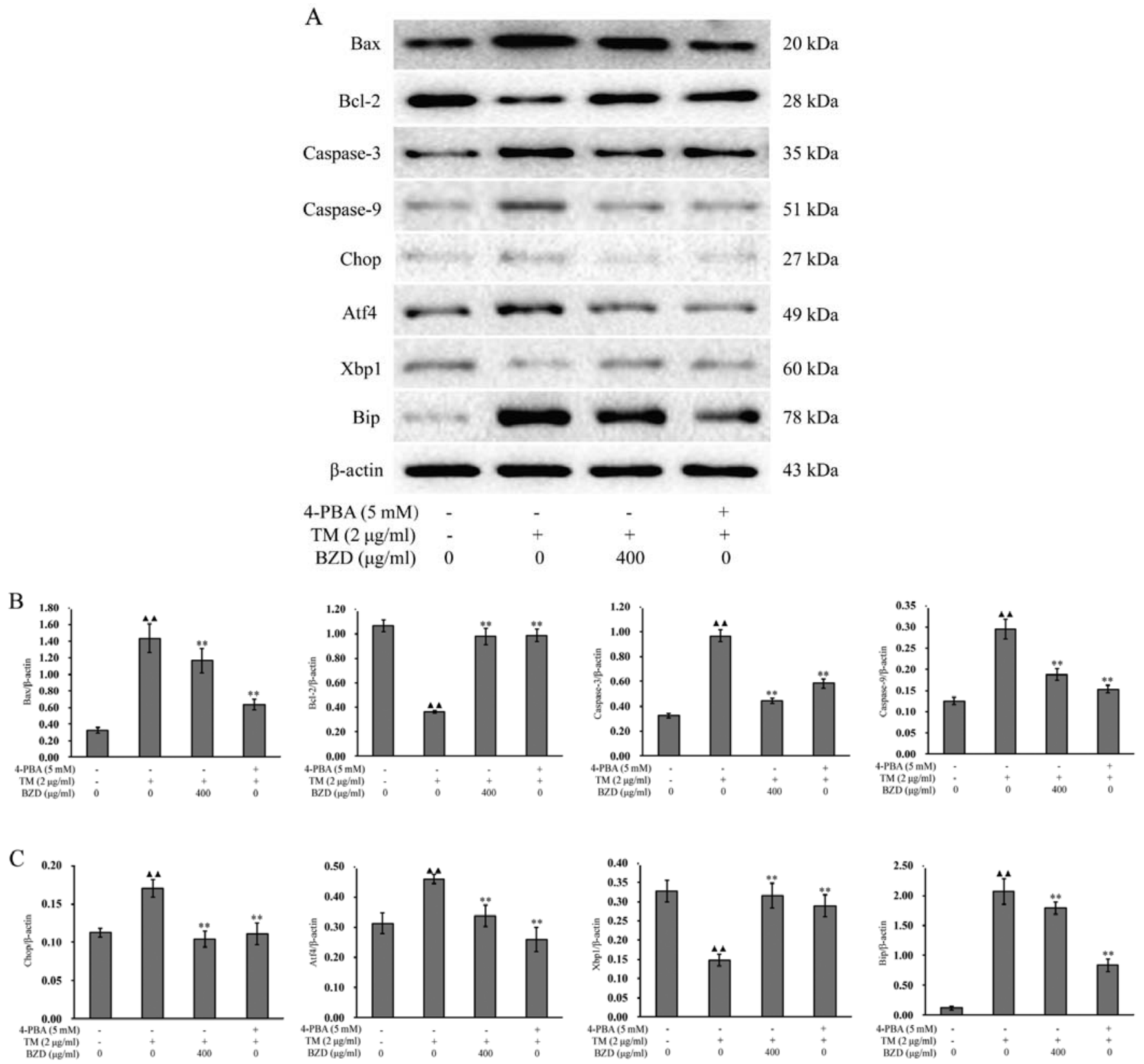

Figure 7. Bushen Zhuangjin decoction (BZD) inhibits the apoptosis of tunicamycin (TM)-stimulated chondrocytes mediated by endoplasmic reticulum (ER) stress. (A-C) Protein expression levels in chondrocytes left untreated and treated with TM and BZD or 4-phenylbutyric acid (4-PBA) in the presence of TM for $48 \mathrm{~h}$ were measured by western blot analysis. The protein expression levels of (C) binding immunoglobin protein (BiP), X-box binding protein 1 (Xbp1), activation transcription factor 4 (Atf4), C/EBP-homologous protein (Chop), (B) Bcl-2-associated X protein (Bax), B-cell lymphoma 2 (Bcl-2), caspase-3 and caspase-9 were measured. $\beta$-actin was used as the internal control for normalization. Values are means $\pm \mathrm{SD}$ (vertical bars). ${ }^{\wedge} \mathrm{P}<0.01$ compared to untreated cells; ${ }^{* *} \mathrm{P}<0.01$, compared to TM-stimulated chondrocytes.

tive effect on TM-stimulated chondrocytes as it inhibits the reduction of $\Delta \Psi \mathrm{m}$ in these cells.

BZD inhibits ER stress-mediated chondrocyte apoptosis. To confirm the inhibitory effects of BZD on ER stress-mediated chondrocyte apoptosis, we measured the expression levels of established the ER stress-associated markers (24), Bip, Xbp1, Chop and Atf4. When ER stress is unresolved, the cell is functionally compromised and may undergo apoptosis (25). Some pathways have been directly implicated in ER stress-induced apoptosis, such as those involving caspases, the Bcl-2 family and the pro-apoptotic member, Bax $(26,27)$. In order to further eludicate the mechanisms responsible for the inhibitory effects of BZD on the apoptosis of TM-stimulated chondrocytes, the mRNA and protein expression levels of apoptosis-associated markers were measured by RT-PCR and western blot analysis, respectively. As shown by the results of RT-PCR, when compared with the model group, the mRNA expression of Bip, Atf4, Chop, Bax, caspase-3 and caspase-9 in the dosing group was downregulated, whereas the expression of Xbp1 and Bcl-2 was upregulated (Fig. 6). Furthermore, the changes in the expression levels of all the aforementioned markers in the dosing group showed no significant differences compared to those observed in the positive control 
group $(\mathrm{P}<0.01, \mathrm{P}<0.05$; Fig. 6$)$. The results obtained for the protein levels were very similar $(\mathrm{P}<0.01, \mathrm{P}<0.05$; Fig. 7$)$. This suggests that BZD inhibits the apoptosis of TM-stimulated chondrocytes by regulating the ER stress pathway.

\section{Discussion}

Increasing evidence indicates that there is an association between cartilage degradation and chondrocyte apoptosis $(28,29)$. Therefore, the inhibition of chondrocyte apoptosis is essential and may be a potential therapeutic strategy for OA. In the present study, we investigated the effects of BZD on TM-induced chondrocyte apoptosis mediated by ER stress. Our results demonstrated that BZD inhibited the apoptosis of TM-stimulated chondrocytes by regulating the ER stress signaling pathway, as evidenced by the upregulation of the expression of Xbp1 and Bcl-2 and the downregulation of the expression of Bip, Atf4, Chop, Bax, caspase- 3 and caspase-9. Currently, pharmacological approaches to the treatment of OA include analgesics, anti-inflammatory agents, intra-articular corticosteroids and hyaluronic acid (30). However, several adverse effects have been associated with these drugs (31-33). Thus, there has been a growing interest in Chinese herbal medicines as they have the same benefits, and are less costly and have fewer adverse effects (34-36). BZD has a good structure and exhibits diverse physicochemical properties. Moreover, it exerts potentially drug-like and multi-target effects $(37,38)$. There is evidence that BZD is effective in the treatment of OA in both clinical and in in vitro studies $(16,17)$.

ER stress activates a set of signaling pathways collectively known as the unfolded protein response (UPR) (39). TM is a bacterial toxin that has been shown to inhibit the N-linked glycosylation of nascent proteins and to lead to the activation of UPR in mammalian cells (40). It is generally used as an ER stress inducer. Thus, TM-stimulated chondrocytes were used as a cellular model of apoptosis in the present study. It was observed that the morphological changes of the TM-stimulated chondrocytes were identical to those accompanying apoptosis, which indicated that the model of apoptosis was successfully established. Additionally, 4-PBA is a chemical chaperone whose beneficial effects have been associated with the suppressed expression of ER stress markers (41). Hence, the TM-stimulated chondrocytes treated with 4-PBA were used as the positive control.

MTT assay is used to monitor cytotoxicity, proliferation and activation (42). In our study, the viability of both untreated and treated cells was measured by MTT assay. According to the results, the viability of the TM-stimulated chondrocytes was evidently enhanced by BZD in a dose- and time-dependent manner. To further examine the effects of BZD on the apoptosis of TM-stimulated chondrocytes, DAPI, Annexin V-FITC binding assay and JC-1 assay were performed. Annexins, the $\mathrm{Ca}^{2+}$-dependent and phospholipid-binding proteins, specifically bind to phosphatidylserine (PS) in the plasma membrane (43). When apoptosis occurs, PS is translocated to the extracellular layer from the cytosolic layer of the plasma membrane, and Annexin V-TITC binding assay is used to detect PS exposure on the membranes of apoptotic cells (44). JC-1 assay detects the loss of $\Delta \Psi \mathrm{m}$. Alterations in $\Delta \Psi \mathrm{m}$ are indicative of changes in the mitochondrial metabolic activity leading to cell cycle arrest and apoptosis; therefore, $\Delta \Psi \mathrm{m}$ is an indicator of cell integrity (45). The results of the Annexin V and JC-1 assays further established the occurrence of apoptosis and demonstrated that BZD reduced the loss of $\Delta \Psi \mathrm{m}$, thereby inhibiting chondrocyte apoptosis.

ER stress is caused by the accumulation of unfolded proteins. Bip maintains the non-activated state of signal transduction factors while combining the end of PERK, Atf6 and IRE1. Xbp1 mRNA, a transcription factor, is frame switched by the removal of a small intron (46). It has been found that ER stress is induced during chondrocyte differentiation and activates the IRE1-Xbp1 pathway (47). ER membrane-associated initiator caspases, such as caspase-3, are also activated by IRE1 $\alpha$ (12). PERK makes a crucial contribution to enhancing the expression of Chop in the UPR, subsequently inducing the translational upregulation of Atf4 by transmitting signals phosphorylating eIF-2a $(48,49)$.

Although apoptosis can be induced by several different triggers, it is characteristically associated downstream with the sequential activation of caspases (50). The caspase family of enzymes is a key factor in cell apoptosis. Caspase-9 is a member of the caspase family of cysteine proteases, which has been implicated in apoptosis and cytokine processing (51). Caspase- 3 is one of the effector caspases that mediates the final stage of cell death by apoptosis (52). It was previously found that the expression of caspase- 3 was $\sim 2$-fold higher in OA-affected cartilage and correlated with chondrocyte apoptosis, activated by caspase-9 (53). Bax (pro-apoptotic molecule) and Bcl-2 (anti-apoptotic molecule) are two biomarkers which are associated with the mechanisms of cell death by apoptosis in OA. It has been demonstrated that Bax is upregulated in OA cartilage and that Bcl-2 is downregulated (54). Bcl-2 is a cytoplasmic protein that is involved in promoting cell survival and preventing apoptosis (55). The transcription of $\mathrm{Bcl}-2$ is regulated by Chop; thus, the upregulation of Bcl-2 may occur during cartilage repair in OA. In our study, the changes in the mRNA and protein levels of these markers were detected by RT-PCR and western blot analysis, respectively. On the one hand, treatment with BZD led to the downregulation of the expression of Bip, Atf4, Chop, Bax, caspase-3 and caspase-9 in the dosing group, thus decreasing chondrocyte apoptosis. On the other hand, BZD upregulated the expression levels of Xbp1 and Bcl-2. Taken together, our findings demonstrated that BZD inhibited the apoptosis of TM-stimulated chondrocytes by regulating the ER stress pathway.

In conclusion, our data demonstrate that BZD effectively exerted its anti-apoptotic effects on chondrocytes stimulated with TM. The inhibition or prevention of chondrocyte apoptosis was mainly the result of the suppression of the ER stress pathway. The findings of the present study indicate that BZD is a potent therapeutic agent for the treatment of OA. However, due to the limitations of in vitro experiments, the exact mechanisms responsible for the inhibition of ER stress-mediated chondrocyte apoptosis by BZD were not completely elucidated. Thus, further studies are warranted to examine the effects of BZD on OA both in vitro and in vivo.

\section{Acknowledgements}

The present study was supported by the National Natural Science Foundation of China (grant no. 81373818), the Key Project of Fujian Provincial Department of Science and 
Technology Department (grant no. 2014Y0064), the Natural Science Foundation of Fujian Province (grant no. 2014J01357), the Special Research Fund for Doctor Discipline in College (grant no. 20123519110001) and the Developmental Fund of Chen Keji Integrative Medicine (grant no. CKJ 2015010).

\section{References}

1. Chu CR, Millis MB and Olson SA: Osteoarthritis: from palliation to prevention: AOA critical issues. J Bone Joint Surg Am 96: e130, 2014

2. Loeser RF: Aging and osteoarthritis: the role of chondrocyte senescence and aging changes in the cartilage matrix. Osteoarthritis Cartilage 17: 971-979, 2009

3. Del Carlo M Jr and Loeser RF: Cell death in osteoarthritis. Curr Rheumatol Rep 10: 37-42, 2008.

4. Kim HA, Lee YJ, Seong SC, Choe KW and Song YW: Apoptotic chondrocyte death in human osteoarthritis. J Rheumatol 27 : 455-462, 2000.

5. Thomas CM, Fuller CJ, Whittles CE and Sharif M: Chondrocyte death by apoptosis is associated with the initiation and severity of articular cartilage degradation. Int J Rheum Dis 14: 191-198, 2011.

6. Hashimoto S, Ochs RL, Komiya S and Lotz M: Linkage of chondrocyte apoptosis and cartilage degradation in human osteoarthritis. Arthritis Rheum 41: 1632-1638, 1998.

7. Almonte-Becerril M, Navarro-Garcia F, Gonzalez-Robles A, Vega-Lopez MA, Lavalle C and Kouri JB: Cell death of chondrocytes is a combination between apoptosis and autophagy during the pathogenesis of Osteoarthritis within an experimental model. Apoptosis 15: 631-638, 2010.

8. Han X, Zhang P, Jiang R, Xia F, Li M and Guo FJ: Explore on the effect of ATF6 on cell growth and apoptosis in cartilage development. Histochem Cell Biol 142: 497-509, 2014.

9. Uehara Y, Hirose J, Yamabe S, Okamoto N, Okada T, Oyadomari S and Mizuta $\mathrm{H}$ : Endoplasmic reticulum stress-induced apoptosis contributes to articular cartilage degeneration via C/EBP homologous protein. Osteoarthritis Cartilage 22: 1007-1017, 2014

10. Ron D and Walter P: Signal integration in the endoplasmic reticulum unfolded protein response. Nat Rev Mol Cell Biol 8: 519-529, 2007.

11. Huang L, Xie $\mathrm{H}$ and Liu H: Endoplasmic reticulum stress, diabetes mellitus, and tissue injury. Curr Protein Pept Sci 15: 812-818, 2014.

12. Oyadomari S and Mori M: Roles of CHOP/GADD153 in endoplasmic reticulum stress. Cell Death Differ 11: 381-389, 2004.

13. Urano F, Wang X, Bertolotti A, Zhang Y, Chung P, Harding HP and Ron D: Coupling of stress in the ER to activation of JNK protein kinases by transmembrane protein kinase IRE1. Science 287: 664-666, 2000.

14. Matsuo M, Nishida K, Yoshida A, Murakami T and Inoue H: Expression of caspase- 3 and -9 relevant to cartilage destruction and chondrocyte apoptosis in human osteoarthritic cartilage. Acta Med Okayama 55: 333-340, 2001.

15. Takada K, Hirose J, Senba K, Yamabe S, Oike Y, Gotoh T and Mizuta H: Enhanced apoptotic and reduced protective response in chondrocytes following endoplasmic reticulum stress in osteoarthritic cartilage. Int J Exp Pathol 92: 232-242, 2011.

16. Zhou GS, Li XF and Guan GH: Effects of Bushen Zhuangjin Decoction containing serum on the apoptosis of chondrocytes induced by mechanics stimulus. Zhongguo Zhong Xi Yi Jie He Za Zhi 32: 789-792, 2012 (In Chinese).

17. Li X, Chen J, Liang W, Li H, Liu F, Weng X, Lin P, Chen W, Zheng $\mathrm{C}, \mathrm{Xu} \mathrm{H}$, et al: Bushen Zhuangjin Decoction promotes chondrocyte proliferation by stimulating cell cycle progression. Exp Ther Med 9: 839-844, 2015.

18. Li X, Liang W, Dang C, et al: Empirical study on Bushen Zhuangjin Decoction inhibiting inflammatory cytokine expression experiments to delay the degeneration of articular cartilage. Feng Shi Bing Yu Guan Jie Yan 3: 20-25, 2014 (In Chinese).

19. Li H, Li X, Liu G, Chen J, Weng X, Liu F, Xu H, Liu X and Ye H: Bauhinia championi (Benth.) Benth. polysaccharides upregulate Wnt/ $\beta$-catenin signaling in chondrocytes. Int J Mol Med 32: 1329-1336, 2013

20. Zeng W, Guo YH, Qi W, Chen JG, Yang LL, Luo ZF, Mu J and Feng B: 4-Phenylbutyric acid suppresses inflammation through regulation of endoplasmic reticulum stress of endothelial cells stimulated by uremic serum. Life Sci 103: 15-24, 2014.
21. Weng X, Lin P, Liu F, Chen J, Li H, Huang L, Zhen C, Xu H, Liu X, Ye H and Li X: Achyranthes bidentata polysaccharides activate the $\mathrm{Wnt} / \beta$-catenin signaling pathway to promote chondrocyte proliferation. Int J Mol Med 34: 1045-1050, 2014.

22. Yu F, Li X, Cai L, Li H, Chen J, Wong X, Xu H, Zheng C, Liu X and $\mathrm{Ye} \mathrm{H}$ : Achyranthes bidentata polysaccharides induce chondrocyte proliferation via the promotion of the G1/S cell cycle transition. Mol Med Rep 7: 935-940, 2013.

23. Ishizeki K, Kagiya T, Fujiwara N, Otsu K and Harada H: Expression of osteogenic proteins during the intrasplenic transplantation of Meckel's chondrocytes: a histochemical and immunohistochemical study. Arch Histol Cytol 72: 1-12, 2009.

24. Araki E, Oyadomari S and Mori M: Endoplasmic reticulum stress and diabetes mellitus. Intern Med 42: 7-14, 2003.

25. Rojas C, Pan-Castillo B, Valls C, Pujadas G, Garcia-Vallve S, Arola L and Mulero M: Resveratrol enhances palmitate-induced ER stress and apoptosis in cancer cells. PLoS One 9: e113929, 2014.

26. Riedl SJ and Shi Y: Molecular mechanisms of caspase regulation during apoptosis. Nat Rev Mol Cell Biol 5: 897-907, 2004.

27. McCullough KD, Martindale JL, Klotz LO, Aw TY and Holbrook NJ: Gadd153 sensitizes cells to endoplasmic reticulum stress by down-regulating $\mathrm{Bcl} 2$ and perturbing the cellular redox state. Mol Cell Biol 21: 1249-1259, 2001.

28. Kim HT, Lo MY and Pillarisetty R: Chondrocyte apoptosis following intraarticular fracture in humans. Osteoarthritis Cartilage 10: 747-749, 2002.

29. D'Lima DD, Hashimoto S, Chen PC, Colwell CW Jr and Lotz MK: Human chondrocyte apoptosis in response to mechanical injury. Osteoarthritis Cartilage 9: 712-719, 2001

30. Tsai CC, Chou YY, Chen YM, Tang YJ, Ho HC and Chen DY: Effect of the herbal drug guilu erxian jiao on muscle strength, articular pain, and disability in elderly men with knee osteoarthritis. Evid Based Complement Alternat Med 2014: 297458, 2014.

31. Gloth FM III: Pharmacological management of persistent pain in older persons: focus on opioids and nonopioids. J Pain 12 (3 Suppl 1): S14-S20, 2011.

32. Schnitzer TJ, Hochberg MC, Marrero CE, Duquesroix B, Frayssinet $\mathrm{H}$ and Beekman $\mathrm{M}$ : Efficacy and safety of naproxcinod in patients with osteoarthritis of the knee: a 53-week prospective randomized multicenter study. Semin Arthritis Rheum 40: 285-297, 2011.

33. Dostrovsky NR, Towheed TE, Hudson RW and Anastassiades TP: The effect of glucosamine on glucose metabolism in humans: a systematic review of the literature. Osteoarthritis Cartilage 19: 375-380, 2011.

34. Lai JN, Chen HJ, Chen CC, Lin JH, Hwang JS and Wang JD: Duhuo jisheng tang for treating osteoarthritis of the knee: a prospective clinical observation. Chin Med 2: 4, 2007.

35. Chen FP, Chang CM, Hwang SJ, Chen YC and Chen FJ: Chinese herbal prescriptions for osteoarthritis in Taiwan: analysis of National Health Insurance dataset. BMC Complement Altern Med 14: 91, 2014.

36. Moudgil KD and Berman BM: Traditional Chinese medicine: potential for clinical treatment of rheumatoid arthritis. Expert Rev Clin Immunol 10: 819-822, 2014.

37. Zheng CS, Ye HZ, Li XH, Xu HF, Wu GW and Liu XX: Study on the active components groups of BUSHENZHUANGJIN decoction for osteoarthritis by molecular docking method. J Tradit Chin Orthop Traumatol 24: 8-10, 2012 (In Chinese).

38. Guo FJ, Xiong Z, Lu X, Ye M, Han X and Jiang R: ATF6 upregulates XBP1S and inhibits ER stress-mediated apoptosis in osteoarthritis cartilage. Cell Signal 26: 332-342, 2014.

39. Bull VH and Thiede B: Proteome analysis of tunicamycin-induced ER stress. Electrophoresis 33: 1814-1823, 2012.

40. Koyama M, Furuhashi M, Ishimura S, Mita T, Fuseya T, Okazaki Y, Yoshida H, Tsuchihashi K and Miura T: Reduction of endoplasmic reticulum stress by 4-phenylbutyric acid prevents the development of hypoxia-induced pulmonary arterial hypertension. Am J Physiol Heart Circ Physiol 306: H1314-H1323, 2014.

41. Mosmann T: Rapid colorimetric assay for cellular growth and survival: application to proliferation and cytotoxicity assays. J Immunol Methods 65: 55-63, 1983.

42. Zhang G, Gurtu V, Kain SR and Yan G: Early detection of apoptosis using a fluorescent conjugate of Annexin V. Biotechniques 23: 525-531, 1997.

43. Dapat E, Jacinto $S$ and Efferth T: A phenolic ester from Aglaia loheri leaves reveals cytotoxicity towards sensitive and multidrug-resistant cancer cells. BMC Complement Altern Med 13: 286, 2013 
44. Acton BM, Jurisicova A, Jurisica I and Casper RF: Alterations in mitochondrial membrane potential during preimplantation stages of mouse and human embryo development. Mol Hum Reprod 10: 23-32, 2004.

45. Zheng C, Ye Z, Li X, Xu H, Wu G and Liu X: Study on diversity and drug-like property of compounds in Bushen Zhuangjin decoction based on computer simulation. Fujian Zhongyi Xueyuan Xuebao 22: 22-25, 2012 (In Chinese).

46. Chevet E, Hetz C and Samali A: Endoplasmic reticulum stressactivated cell reprogramming in oncogenesis. Cancer Discov 5: 586-597, 2015

47. Puthalakath H, O'Reilly LA, Gunn P, Lee L, Kelly PN, Huntington ND, Hughes PD, Michalak EM, McKimm-Breschkin J, Motoyama N, et al: ER stress triggers apoptosis by activating BH3-only protein Bim. Cell 129: 1337-1349, 2007

48. Fu HY, Okada K, Liao Y, Tsukamoto O, Isomura T, Asai M, Sawada T, Okuda K, Asano Y, Sanada S, et al: Ablation of C/EBP homologous protein attenuates endoplasmic reticulum-mediated apoptosis and cardiac dysfunction induced by pressure overload. Circulation 122: 361-369, 2010.

49. B'chir W, Maurin AC, Carraro V, Averous J, Jousse C, Muranishi Y, Parry L, Stepien G, Fafournoux P and Bruhat A: The eIF2 $\alpha /$ ATF4 pathway is essential for stress-induced autophagy gene expression. Nucleic Acids Res 41: 7683-7699, 2013.
50. Creagh EM: Caspase crosstalk: integration of apoptotic and innate immune signalling pathways. Trends Immunol 35: 631-640, 2014

51. Allan LA and Clarke PR: Apoptosis and autophagy: Regulation of caspase-9 by phosphorylation. FEBS J 276: 6063-6073, 2009.

52. Jänicke RU, Sprengart ML, Wati MR and Porter AG: Caspase-3 is required for DNA fragmentation and morphological changes associated with apoptosis. J Biol Chem 273: 9357-9360, 1998.

53. Sharif M, Whitehouse A, Sharman P, Perry M and Adams M: Increased apoptosis in human osteoarthritic cartilage corresponds to reduced cell density and expression of caspase- 3 . Arthritis Rheum 50: 507-515, 2004.

54. Liu F, Liu G, Liang W, Ye H, Weng X, Lin P, Li H, Chen J, Liu X and Li X: Duhuo Jisheng decoction treatment inhibits the sodium nitroprussiate-induced apoptosis of chondrocytes through the mitochondrial-dependent signaling pathway. Int J Mol Med 34: 1573-1580, 2014.

55. Hildeman DA, Zhu Y, Mitchell TC, Kappler J and Marrack P: Molecular mechanisms of activated T cell death in vivo. Curr Opin Immunol 14: 354-359, 2002. 